\title{
BOUNDARY BEHAVIOR AND PRODUCT-FORM STATIONARY DISTRIBUTIONS OF JUMP DIFFUSIONS IN THE ORTHANT WITH STATE-DEPENDENT REFLECTIONS
}

\author{
FRANCISCO J. PIERA, ${ }^{*}$ University of Chile \\ RAVI R. MAZUMDAR, ${ }^{* *}$ University of Waterloo \\ FABRICE M. GUILLEMIN, ${ }^{* * *}$ France Telecom $R \& D$
}

\begin{abstract}
In this paper we consider reflected diffusions with positive and negative jumps, constrained to lie in the nonnegative orthant of $\mathbb{R}^{n}$. We allow for the drift and diffusion coefficients, as well as for the directions of reflection, to be random fields over time and space. We provide a boundary behavior characterization, generalizing known results in the nonrandom coefficients and constant directions of the reflection case. In particular, the regulator processes are related to semimartingale local times at the boundaries, and they are shown not to charge the times the process expends at the intersection of boundary faces. Using the boundary results, we extend the conditions for product-form distributions in the stationary regime to the case when the drift and diffusion coefficients, as well as the directions of reflection, are random fields over space.
\end{abstract}

Keywords: Diffusion; jumps; reflection map; regulator process; local time; semimartingale; stationary distribution; product form

2000 Mathematics Subject Classification: Primary 60J60; 60J75

Secondary 60J50; 60J55; 60K25

\section{Introduction}

Let $n \geq 2$ be an integer, let $\mathbb{R}$ and $\mathbb{R}_{+}$respectively be the set of real and nonnegative real numbers, let $\mathbb{R}^{n}:=\times_{i=1}^{n} \mathbb{R}$ and $\mathbb{R}_{+}^{n}:=\times_{i=1}^{n} \mathbb{R}_{+}$, and let $\mathbb{R}^{n \times n}$ be the collection of all $n \times n$ real matrices. Throughout the paper, equalities and inequalities involving vectors or vector-valued processes are to be understood componentwise, ' 0 ' represents the appropriate null element clear from the context, ' $\infty$ ' denotes $(\infty, \ldots, \infty)$ in a vectorial context, and, even though vectors or vector-valued processes are written as row vectors, they are treated as column vectors in all the equations in which they appear. In [11] the following reflected diffusion with positive and negative jumps, constrained to lie in $\mathbb{R}_{+}^{n}$, was considered:

$$
X_{t}=X_{0}+\int_{0}^{t} b\left(s, X_{s-}\right) \mathrm{d} t+\int_{0}^{t} \gamma\left(s, X_{s-}\right) \mathrm{d} W_{s}+\sum_{0<s \leq t} \Delta X_{s}+R Z_{t},
$$

\footnotetext{
Received 18 November 2005; revision received 28 March 2008.

* Postal address: Department of EE, University of Chile, Av. Tupper 2007, Santiago, 8370451, Chile.

Email address: fpiera@ing.uchile.cl

** Postal address: Department of ECE, University of Waterloo, Waterloo, ON N2L 3G1, Canada.

Email address: mazum@ece.uwaterloo.ca

*** Postal address: France Telecom R\&D, 2 Avenue Pierre Marzin, 22300 Lannion, France.

Email address: fabrice.guillemin@francetelecom.com
} 
where $X$ is a càdlàg semimartingale (i.e. that which is continuous from the right with left limits) with signed jumps $\Delta X_{t}:=X_{t}-X_{t-}$ satisfying $\sum_{0<s \leq t}\left|\Delta X_{s}\right|<\infty$ almost surely (a.s.) for each $t>0\left(X_{t-}:=\lim _{s \uparrow t} X_{s}\right.$ with $X_{0-}:=0$ by convention $), b$ is the vector of drift coefficients, $\gamma$ is the matrix of dispersion coefficients, $W$ is an $n$-dimensional standard Brownian motion, $R \in \mathbb{R}^{n \times n}$ is the reflection matrix whose columns give the directions of reflection of $X$ upon hitting the boundary faces of $\mathbb{R}_{+}^{n}$, and, finally, $Z$ is the vector of regulator processes keeping $X$ in the nonnegative orthant. In [11] we established a boundary behavior characterization for the tuple $(X, Z)$, showing that the regulators, i.e. the $Z^{i}$ s, do not charge the set of times spent by $X$ at the intersection of two or more boundary faces. In addition, we characterized each $Z^{i}$ in terms of the semimartingale local-time process at level 0 of $X^{i}, L^{i}(\cdot, 0)$, as

$$
Z_{.}^{i}=\frac{L^{i}(\cdot, 0)}{2 R_{i, i}},
$$

where $R_{i, i}>0$ is the $i$ th diagonal element of matrix $R$. All these boundary properties were established in [11] under appropriate mild boundary conditions on the diffusion coefficients $a:=$ $\gamma \gamma^{\top}$ (where $\gamma^{\top}$ denotes the transpose of matrix $\gamma$ ), as well as under a completely-S structure with an additional invertibility requirement on the reflection matrix (completely-S matrices are described in the next section of the paper). A representation as in (2) provides a useful interpretation of $Z$, and gives an alternative characterization over the well-known (implicit) representation $Z^{i}=\left(1 / R_{i, i}\right) \sup _{s \in[0, \cdot]} \max \left\{-V_{s}^{i}, 0\right\}$ with $V^{i}:=X^{i}-R_{i, i} Z^{i}$ (implicit in the sense that $V^{i}$ contains the components $Z^{j}, j \neq i$ ). For the class of reflection matrices of the form $R=I-Q$, where $I$ is the identity matrix and $Q \geq 0$ elementwise with spectral radius less than 1, process $Z$ also results from applying the multidimensional reflection map to the unconstrained or 'free' process $U:=X-R Z$ and can be characterized as follows: $Z$ is the minimal element in the space of continuous processes, null at zero and (componentwise) nondecreasing, such that $U+R Z \geq 0$, i.e. if $Y$ has continuous and nondecreasing paths, and it is such that $Y_{0}=0$ and $U+R Y \geq 0$, then $Z \leq Y$. For the extension to completely-S reflection matrices, see [19] and the references therein. Further discussion on regulator processes and reflection maps can also be found, for example, in [3], [4], [13], [17], [18], and the references therein.

In this paper we will consider (1) in the general case when $b$ and $\gamma$ are in addition allowed to be random, and when the reflection matrix $R$ is an $\mathbb{R}^{n \times n}$-valued random field over time and space as well. We will show that, under the natural extended assumptions, all the previous results in [11] generalize to this case. In particular, we will show that the characterization of $Z$ in (2) comes from the following general representation:

$$
Z_{.}^{i}=\frac{1}{2} \int_{0}^{\cdot} \frac{L^{i}(\mathrm{~d} s, 0)}{R_{i, i}\left(s, X_{s}\right)} .
$$

The detailed model formulation will be provided in the next section of the paper.

An appropriate boundary behavior characterization has been shown to play an important role in studying and characterizing the stationary distribution of a reflected process, when it exists, as well as in establishing when in fact a stationary version exists. For example, in [9] the regulator process of a one-dimensional reflected diffusion with jumps was related to the corresponding semimartingale local time at level zero, and this connection was then used to study the stationary distributions of such processes. Harrison and Williams [5] used the boundary property established in [14], in that the regulator processes do not charge the set of 
times spent by a semimartingale reflecting Brownian motion (SRBM) at the intersection of two or more boundary faces of $\mathbb{R}_{+}^{n}$, to obtain necessary and sufficient conditions for the existence of the stationary regime for such processes, characterizing the case when the stationary distribution is in product form, i.e. it is expressed as the product of its one-dimensional marginals. In [16] the boundary property established in [14] was used in the numerical computation of the stationary distribution of an SRBM in a hypercube. In the context of reflected Lévy processes (including jumps), product-form stationary distributions have been studied, for example, in [1] and [7], where once again an appropriate boundary behavior characterization was used.

Recently, in [10] the boundary behavior characterization provided in [11] was used to obtain forward equations for the stationary density of $X$ in (1), with an appropriate jump measure driving the jumps in $X$ and with $b$ and $\gamma$ not depending on time, of course, and assuming that the stationary setting and a density (with respect to the Lebesgue measure) exist. These equations were then used to establish necessary and sufficient conditions for this density to be in product form, generalizing the previously known product-form conditions for SRBMs in [5] and the corresponding negative results for reflected Lévy processes (including jumps) in [7]. Although, for simplicity, the case of positive jumps were considered in [10], it was indicated there how all the results extend to the case of positive and negative jumps.

In this paper we will also generalize the necessary and sufficient conditions for a productform distribution (in the stationary regime) established in [10] to the case when $b$ and $\gamma$ are in addition allowed to be random, and when the reflection matrix $R$ is an $\mathbb{R}^{n \times n}$-valued random field over space as well. Some specific cases and their related equations will be discussed explicitly.

The motivation to establish a boundary behavior characterization useful in describing the stationary distribution of reflected diffusions with jumps is manyfold. This class of processes has lately found a wide variety of applications in several fields, such as mathematical finance, risk, and queueing theory. For example, in queueing theory and stochastic networks they appear in the context of weak limits under heavy traffic conditions, where complex network models can be approximated in a weak (or in distribution) sense by a reflected diffusion with jumps in the limiting case when the utilizations take values near unity. See, for example, [2], [5], [6], [8], [17]-[20], and the references therein.

The organization of the paper is as follows. In Section 2 we introduce the model to be considered, giving some notation and terminology that will be used throughout. In Section 3 we provide a boundary behavior characterization for the general framework introduced in Section 2 . Finally, in Section 4 we extend to this general framework the necessary and sufficient conditions for a product-form distribution in the stationary regime, considering explicitly some specific cases and their related equations.

\section{Model formulation and further notation}

Let $n \geq 2$ be an integer, and let $\left(\Omega, \mathcal{F},\left(\mathcal{F}_{t}\right)_{t \geq 0}, \mathrm{P}\right)$ be a stochastic basis satisfying the usual hypotheses, i.e. $\mathcal{F}_{0}$ contains all the P-null sets of $\mathscr{F}$ and the filtration $\left(\mathscr{F}_{t}\right)_{t \geq 0}$ is right continuous. (At this point readers may wish to review the notation and terminology introduced at the beginning of Section 1.) We consider the following reflected diffusion with positive and negative jumps, state space $\mathbb{R}_{+}^{n}$, and random, time- and state-dependent drift and diffusion coefficients, as well as random, time- and state-dependent directions of reflection upon hitting 
$\partial_{0}:=\bigcup_{i=1}^{n} \partial_{i}:=\bigcup_{i=1}^{n}\left\{x \in \mathbb{R}_{+}^{n}: x_{i}=0\right\}:$

$X_{t}=X_{0}+\int_{0}^{t} b\left(\omega, s, X_{s-}\right) \mathrm{d} s+\int_{0}^{t} \gamma\left(\omega, s, X_{s-}\right) \mathrm{d} W_{s}+\sum_{0<s \leq t} \Delta X_{s}+\int_{0}^{t} R\left(\omega, s, X_{s-}\right) \mathrm{d} Z_{s}$,

where $\omega \in \Omega$ and the following notation is used.

- $X=\left(X_{t}\right)_{t \geq 0}=\left(X_{t}^{1}, \ldots, X_{t}^{n}\right)_{t \geq 0}$ is an $\left(\mathcal{F}_{t}\right)_{t \geq 0}$-adapted, $\mathbb{R}_{+}^{n}$-valued càdlàg semimartingale. (Recall that $X_{t-}:=\lim _{s \uparrow t} X_{s}$ with $X_{0-}:=0$ and $\Delta X_{t}:=X_{t}-X_{t-}$.) We assume hereafter that $\sum_{0<s \leq t}\left|\Delta X_{s}\right|<\infty$ a.s. for each $t>0$.

- $W=\left(W_{t}\right)_{t \geq 0}=\left(W_{t}^{1}, \ldots, W_{t}^{n}\right)_{t \geq 0}$ is an $\left(\mathcal{F}_{t}\right)_{t \geq 0}$-standard Brownian motion on $\mathbb{R}^{n}$.

- $Z=\left(Z_{t}\right)_{t \geq 0}=\left(Z_{t}^{1}, \ldots, Z_{t}^{n}\right)_{t \geq 0}$ is a continuous, $\left(\widetilde{F}_{t}\right)_{t \geq 0}$-adapted, $\mathbb{R}_{+}^{n}$-valued process with each $Z^{i}$ nondecreasing, null at zero, and such that $\int_{\mathbb{R}_{+}} X_{s}^{i} \mathrm{~d} Z_{s}^{i}=0$.

- $b=\left(b_{i}\right)_{i \in\{1, \ldots, n\}}: \Omega \times \mathbb{R}_{+}^{n+1} \rightarrow \mathbb{R}^{n}$ and $\gamma=\left(\gamma_{i, j}\right)_{i, j \in\{1, \ldots, n\}}: \Omega \times \mathbb{R}_{+}^{n+1} \rightarrow \mathbb{R}^{n \times n}$ are random fields with $\mathcal{F} \otimes \mathscr{B}\left(\mathbb{R}_{+}^{n+1}\right)-\mathscr{B}(\mathbb{R})$ measurable coefficients, both $\left(\mathcal{F}_{t}\right)_{t \geq 0}$-adapted for each fixed $x \in \mathbb{R}_{+}^{n}$. (Recall that we set $a=\left(a_{i, j}\right)_{i, j \in\{1, \ldots, n\}}:=\gamma \gamma^{\top}$.)

- $R=\left(R_{i, j}\right)_{i, j \in\{1, \ldots, n\}}: \Omega \times \mathbb{R}_{+}^{n+1} \rightarrow \mathbb{R}^{n \times n}$ is a random field with $\mathcal{F} \otimes \mathscr{B}\left(\mathbb{R}_{+}^{n+1}\right)-\mathscr{B}(\mathbb{R})$ measurable coefficients, $\left(\mathcal{F}_{t}\right)_{t \geq 0}$-adapted for each fixed $x \in \mathbb{R}_{+}^{n}$. Note that the definition of $R$ is only relevant for $(\omega, t, x) \in \Omega \times \mathbb{R}_{+} \times \partial_{0}$; since $X$ is càdlàg and $Z$ is continuous, we have $\int_{0}^{\cdot} R_{i, j}\left(s, X_{s-}\right) \mathrm{d} Z_{s}^{j}=\int_{0}^{\cdot} R_{i, j}\left(s, X_{s}\right) \mathrm{d} Z_{s}^{j}, i, j \in\{1, \ldots, n\}$ (see Remark 1 , below), and, moreover, each $Z^{j}$ can only increase at times $s$ when $X_{s}^{j}=0$ (i.e. when $\left.X_{s} \in \partial_{j}\right)$, by definition of $Z$. In addition, we assume that $\mathrm{P}\left\{\omega \in \Omega: R(\omega, t, x) \in \mathcal{R}_{n}\right.$ for all $\left.(t, x) \in \mathbb{R}_{+} \times \partial_{0}\right\}=1$, where $\mathcal{R}_{n}$ denotes the collection of all $n \times n$ completely-S matrices (described below) with the additional property that each of their principal submatrices is nonsingular, i.e. $D \in \mathcal{R}_{n}$ if $D$ is an $n \times n$ completely-S matrix and, for each $K \subsetneq\{1, \ldots, n\}$, the principal submatrix obtained from $D$ by deleting its $k$ th row and column for all $k \in K$ (none if $K=\varnothing$ ), denoted as $D^{(K)}$, is invertible.

Recall that a matrix $D$ in $\mathbb{R}^{n \times n}$ is said to be completely-S if it has the property that, for each $\tilde{D}$ principal submatrix of $D$, there exists a vector $y \geq 0$, of the corresponding proper dimension, such that $\tilde{D} y>0$. (Note in particular that $D$ must have strictly positive diagonal elements.) In [14] the authors showed the necessity of this condition on the (constant, nonrandom) reflection matrix of SRBMs in the nonnegative orthant for such processes to exist, which accounts for the fact that, upon hitting the boundary of $\mathbb{R}_{+}^{n}$, the process cannot leave the orthant.

As in [11], the additional invertibility requirement on $R$ will allow us to relate the regulator processes $Z^{i}$ s to semimartingale local times, as well as to establish the boundary property in that the $Z^{i}$ s do not charge the set of times spent by $X$ at the intersection of two or more boundary faces. Examples of matrices in $\mathcal{R}_{n}$ are P-matrices, i.e. matrices for which each principal minor is strictly positive, as, for example, positive definite matrices, real triangular matrices with strictly positive diagonal elements, and matrices of the form $s_{0} I-Q$ with $s_{0}>0$, where $I$ is the identity matrix and $Q \geq 0$ elementwise with spectral radius strictly less than $s_{0}$.

In this paper we assume the existence of a tuple $(X, Z)$, as described, satisfying (3), i.e. with semimartingale $X-X_{0}$ having canonical decomposition $X-X_{0}=A+M+J, A_{0}=M_{0}=$ $J_{0}:=0$, where the continuous finite-variation process $A$ and the continuous local martingale term $M$ are given by $A .:=\int_{0}^{\cdot} b\left(s, X_{s-}\right) \mathrm{d} s+R Z$. and $M .:=\int_{0}^{\cdot} \gamma\left(s, X_{s-}\right) \mathrm{d} W_{s}$, and where $J .:=\sum_{0<s \leq .} \Delta X_{s}$ with $\sum_{0<s \leq t}\left|\Delta X_{s}\right|<\infty$ a.s. for each $t>0$. It is not the aim of the paper 
to deal with existence issues. Results on existence and uniqueness of solutions to reflecting stochastic differential equations with random coefficients and jumps driven by Poisson random measures (with jump amplitudes expressed as functions of $X_{t-}$ ) can be found in [15].

We now introduce some notation that will be used throughout. For integers $k, l \geq 1$, we denote by $\mathcal{C}^{l}\left(\mathbb{R}_{+}^{k}\right)$ the space of all functions $f: \mathbb{R}_{+}^{k} \rightarrow \mathbb{R}$ with continuous partial derivatives up to and including order $l$, and we denote by $\mathcal{C}_{b}^{l}\left(\mathbb{R}_{+}^{k}\right)$ the subspace of functions in $\mathcal{C}^{l}\left(\mathbb{R}_{+}^{k}\right)$ that, along with all their partial derivatives up to and including order $l$ are bounded. As usual, when no superscript $l$ is written we refer to the functions themselves, excluding their derivatives. In addition, for $f: \mathbb{R}_{+}^{k} \rightarrow \mathbb{R}$ and $i \in\{1, \ldots, k\}$, we write $f\left(0_{i}\right)$ to indicate that the $i$ th argument in $f$ is set to $0, f\left(0_{i}+\right)$ to denote $\lim _{x_{i} \downarrow 0} f(x)$, and $\mathcal{L}_{k}[f](\cdot)$ to denote the (k-dimensional) Laplace transform of $f$ (omitting $i$ and $k$ when $f$ is defined over $\mathbb{R}_{+}$). Also, we write $P_{t}^{X}$ for the law of $X_{t}$ in $\mathbb{R}_{+}^{n}, t \in \mathbb{R}_{+}$, and $\left(L^{i}(t, r)\right)_{t, r \geq 0}$ for the jointly continuous in $t$ and right continuous in $r$ version of the local time ( $r$ indicating the level) associated with semimartingale $X^{i}$. Note this version exists and, moreover, for every $(t, r) \in \mathbb{R}_{+}^{2}$, we have

$$
L^{i}(t, r)=\lim _{\varepsilon \downarrow 0} \frac{1}{\varepsilon} \int_{0}^{t} \mathbf{1}\left\{r \leq X_{s}^{i} \leq r+\varepsilon\right\} a_{i, i}\left(s, X_{s-}\right) \mathrm{d} s \quad \text { a.s. }
$$

( $X$ being a càdlàg semimartingale satisfying $\sum_{0<s<t}\left|\Delta X_{s}\right|<\infty$ a.s. for each $t>0$ (see [12, Theorem 56 and Corollary 3])). It is obviously enough for us to consider here only $r \in \mathbb{R}_{+}$, since $X$ is constrained to lie in $\mathbb{R}_{+}^{n}$. In addition, for $K, \tilde{K} \subseteq\{1, \ldots, n\}, K \backslash \tilde{K}$ denotes the usual set-theoretic difference and $|K|$ denotes the number of elements in $K$. Also, $m$ and $\otimes_{n} m$ denote the Lebesgue measures in $\mathbb{R}_{+}$and in $\mathbb{R}_{+}^{n}$, respectively. Finally, we set $\partial_{i}^{\circ}:=\partial_{i} \backslash \bigcup_{k=1, k \neq i}^{n} \partial_{k}$, and denote by $\mathrm{d} x_{\neq i}$ the differential $\mathrm{d} x_{1} \cdots \mathrm{d} x_{n}$ when $\mathrm{d} x_{i}$ is omitted and denote by $\mathbf{1}\{\cdot\}$ the indicator function of the corresponding event in parentheses.

As we have done so far, whenever we write a.s., it is with respect to P. Similarly, in what follows, whenever we write a.e. (almost every or almost everywhere), it is with respect to the Lebesgue measure in the corresponding real space (clear from the context).

Finally, we make the following remark.

Remark 1. Since $X$ is càdlàg, it can have at most a countable number of jumps in any compact interval of times contained in $\mathbb{R}_{+}$. Therefore, $X_{s-}$ can always be replaced by $X_{s}$ in integrals of the form $\int_{0}^{\cdot} f\left(X_{s-}\right) \mu(\mathrm{d} s)$, and vice versa, when the measure $\mu$ is diffuse, i.e. when $\mu$ has no atoms. Note that this is the case for the Lebesgue measure and for the random measures $\mathrm{d} Z_{s}^{i}$ and $L^{i}(\mathrm{~d} s, r)$ that $Z_{s}^{i}$ and $L^{i}(s, r)$ induce in $\mathbb{R}_{+}$, respectively. This fact will be used from now on without further comment.

\section{Boundary behavior characterization}

In this section we obtain a boundary behavior characterization for $(X, Z)$ in (3). The results in this section generalize the corresponding ones in [11], where $b$ and $\gamma$ are nonrandom, and where $R$ is a constant matrix in $\mathcal{R}_{n}$. The proofs given here focus on the points of difference between the corresponding proofs given in [11].

Lemma 1. Let $t \in \mathbb{R}_{+}$, and let $K \subseteq\{1, \ldots, n\}$. Assume that there exists $i \in K$ such that $\mathrm{P}\left\{\omega \in \Omega: a_{i, i}(\omega, s, x)>0\right.$ for all $\left.x \in \bigcap_{k \in K} \partial_{k}\right\}=1$ for a.e. $s \in[0, t]$. Then, we have

$$
m\left\{s \in[0, t]: X_{s} \in \bigcap_{k \in K} \partial_{k}\right\}=0 \quad \text { a.s. }
$$


Also, for a.e. $s \in[0, t]$,

$$
P_{s}^{X}\left\{\bigcap_{k \in K} \partial_{k}\right\}=0
$$

i.e. for a.e. $s \in[0, t], P_{s}^{X}$ does not charge the set $\bigcap_{k \in K} \partial_{k}$.

Proof. Follows by the same arguments as in the proof of Lemma 2.1 of [11].

Remark 2. When $K=\{i\} \subseteq\{1, \ldots, n\}$, under the assumptions in Lemma 1 , we have $P_{s}^{X}\left\{\partial_{i}\right\}=0$ for a.e. $s \in[0, t]$.

For the next lemma, we require some preliminary elements. Let $i \in\{1, \ldots, n\}$, let $r \in \mathbb{R}_{+}$, and let $\Psi=(\Psi(\omega, x)): \Omega \times \mathbb{R}_{+}^{n} \rightarrow \mathbb{R}$ be an $\mathcal{F}_{0} \otimes \mathscr{B}\left(\mathbb{R}_{+}^{n}\right)-\mathscr{B}(\mathbb{R})$ measurable random field. Furthermore, let us write $\sigma_{t}$ for the $\sigma$-algebra generated by $X_{t}$, and write $X_{t}^{r_{i}}$ for $X_{t}$ when its $i$ th component $X_{t}^{i}$ is replaced by $r$, i.e. $X_{t}^{r_{i}}:=\left(X_{t}^{1}, \ldots, X_{t}^{i-1}, r, X_{t}^{i+1}, \ldots, X_{t}^{n}\right)$. Then, since $|\Psi| a_{i, i} \geq 0$, we conclude that there exists $h_{|\Psi|, r}^{i}: \mathbb{R}_{+}^{n+1} \rightarrow \mathbb{R}_{+} \cup\{\infty\}$, Borel measurable, such that $h_{|\Psi|, r}^{i}\left(t, X_{t}(\omega)\right)=\mathrm{E}\left[\left|\Psi\left(X_{t}^{r_{i}}\right)\right| a_{i, i}\left(t, X_{t}\right) \mid \sigma_{t}\right](\omega)$ a.s. for each $t \in \mathbb{R}_{+}$, and note that

$$
\int_{0}^{t} \mathrm{E}\left[\left|\Psi\left(X_{s}^{r_{i}}\right)\right| a_{i, i}\left(s, X_{s}\right)\right] \mathrm{d} s=\int_{0}^{t} \int_{\mathbb{R}_{+}^{n}} h_{|\Psi|, r}^{i}(s, x) P_{s}^{X}(\mathrm{~d} x) \mathrm{d} s
$$

for each $t \in \mathbb{R}_{+}$(both being finite or $\infty$ ). Moreover, note that if, for some $t \in \mathbb{R}_{+}$, we have $\mathrm{E}\left[\left|\Psi\left(X_{s}^{r_{i}}\right)\right| a_{i, i}\left(s, X_{s}\right)\right]<\infty$ for a.e. $s \in[0, t]$ then there exists $N_{t} \subset[0, t], m\left\{N_{t}\right\}=0$, such that the conditional expectation $\mathrm{E}\left[\Psi\left(X_{s}^{r_{i}}\right) a_{i, i}\left(s, X_{s}\right) \mid \sigma_{s}\right](\omega)$ is well defined and finite a.s. for each $s \in[0, t] \backslash N_{t}$. Setting it as any real value for each $s \in N_{t}$ and all $\omega \in \Omega$, we conclude that there exists $h_{\Psi, r}^{i}:[0, t] \times \mathbb{R}_{+}^{n} \rightarrow \mathbb{R}$, Borel measurable, such that $h_{\Psi, r}^{i}\left(s, X_{s}(\omega)\right)=$ $\mathrm{E}\left[\Psi\left(X_{s}^{r_{i}}\right) a_{i, i}\left(s, X_{s}\right) \mid \sigma_{s}\right](\omega)$ a.s. for each $s \in[0, t]$, and note that, with this construction,

$$
\int_{0}^{t} \mathrm{E}\left[\Psi\left(X_{s}^{r_{i}}\right) a_{i, i}\left(s, X_{s}\right)\right] \mathrm{d} s=\int_{0}^{t} \int_{\mathbb{R}_{+}^{n}} h_{\Psi, r}^{i}(s, x) P_{s}^{X}(\mathrm{~d} x) \mathrm{d} s
$$

if $\int_{0}^{t} \mathrm{E}\left[\left|\Psi\left(X_{s}^{r_{i}}\right)\right| a_{i, i}\left(s, X_{s}\right)\right] \mathrm{d} s<\infty$. Finally, for each $\varepsilon \geq 0$, we write $\mathbb{R}_{+}^{n}(i, r, \varepsilon)$ for the set $\left\{x \in \mathbb{R}_{+}^{n}: r \leq x_{i} \leq r+\varepsilon\right\}$.

Now we can state the lemma. Even though we will require it in this section for nonrandom $\Psi$ (i.e. independent of $\omega \in \Omega$ ), its corollary, Corollary 1, below, will be required for random $\Psi$ in Subsection 4.2.

Lemma 2. Let $t, r \in \mathbb{R}_{+}$, let $i \in\{1, \ldots, n\}$, and let $\Psi=(\Psi(\omega, x)): \Omega \times \mathbb{R}_{+}^{n} \rightarrow \mathbb{R}$ be a bounded random field, measurable as above. Assume that there exists $\eta>0$ for which

$$
\int_{0}^{t} \int_{\mathbb{R}_{+}^{n}(i, r, \eta)} h_{|\Psi|, r}^{i}(s, x) P_{s}^{X}(\mathrm{~d} x) \mathrm{d} s<\infty .
$$

Then, with $\Psi_{\eta}(\cdot, x)=\Psi(\cdot, x)$ if $x \in \mathbb{R}_{+}^{n}(i, r, \eta)$ and $\Psi_{\eta}(\cdot, x)=0$ elsewhere, we have

$$
\mathrm{E}\left[\int_{0}^{t} \Psi\left(X_{S}\right) L^{i}(\mathrm{~d} s, r)\right]=\lim _{\varepsilon \downarrow 0} \frac{1}{\varepsilon} \int_{0}^{t} \int_{\mathbb{R}_{+}^{n}(i, r, \varepsilon)} h_{\Psi_{\eta}, r}^{i}(s, x) P_{s}^{X}(\mathrm{~d} x) \mathrm{d} s .
$$

Proof. Since, for each $\varepsilon \in \mathbb{R}_{+}$,

$$
\int_{0}^{t} \mathrm{E}\left[\left|\Psi\left(X_{s}^{r_{i}}\right)\right| \mathbf{1}\left\{r \leq X_{s}^{i} \leq r+\varepsilon\right\} a_{i, i}\left(s, X_{s}\right)\right] \mathrm{d} s=\int_{0}^{t} \int_{\mathbb{R}_{+}^{n}(i, r, \varepsilon)} h_{|\Psi|, r}^{i}(s, x) P_{s}^{X}(\mathrm{~d} x) \mathrm{d} s
$$


and the right-hand side of the above equation is finite for $\varepsilon=\eta$ and, hence, for each $\varepsilon \in[0, \eta]$ $\left(h_{|\Psi|, r}^{i} \geq 0\right)$, we conclude that

$$
\int_{0}^{t} \mathrm{E}\left[\Psi\left(X_{s}^{r_{i}}\right) \mathbf{1}\left\{r \leq X_{s}^{i} \leq r+\varepsilon\right\} a_{i, i}\left(s, X_{s}\right)\right] \mathrm{d} s=\int_{0}^{t} \int_{\mathbb{R}_{+}^{n}(i, r, \varepsilon)} h_{\Psi_{\eta}, r}^{i}(s, x) P_{s}^{X}(\mathrm{~d} x) \mathrm{d} s
$$

for each $\varepsilon \in[0, \eta]$. Now, by the same arguments as in the proof of Lemma 2.2 of [11], and by using Fubini's theorem, we conclude that, for each $\varepsilon \in(0, \eta]$,

$$
\frac{1}{\varepsilon} \int_{r}^{r+\varepsilon} \mathrm{E}\left[\int_{0}^{t} \Psi\left(X_{s}^{r_{i}}\right) L^{i}(\mathrm{~d} s, u)\right] \mathrm{d} u=\frac{1}{\varepsilon} \int_{0}^{t} \mathrm{E}\left[\Psi\left(X_{s}^{r_{i}}\right) \mathbf{1}\left\{r \leq X_{s}^{i} \leq r+\varepsilon\right\} a_{i, i}\left(s, X_{s}\right)\right] \mathrm{d} s .
$$

The proof of the lemma now follows as in the proof of Lemma 2.2 of [11].

Corollary 1. Let $t, r \in \mathbb{R}_{+}$, let $i \in\{1, \ldots, n\}$, and let $\Psi=(\Psi(\omega, x)): \Omega \times \mathbb{R}_{+}^{n} \rightarrow \mathbb{R}$ be a bounded random field, measurable as above. Assume that $P_{s}^{X}(\mathrm{~d} x)$ admits a (jointly measurable in $s$ and $x$ ) density $p_{X, s}(x)$ (with respect to the Lebesgue measure), and that there exists $\eta>0$ for which

$$
\int_{0}^{t} \int_{\mathbb{R}_{+}^{n-1}} \sup _{r \leq x_{i} \leq r+\eta}\left\{h_{|\Psi|, r}^{i}(s, x) p_{X, s}(x)\right\} \mathrm{d} x_{\neq i} \mathrm{~d} s<\infty .
$$

Furthermore, assume that, for a.e. $s \in[0, t], \lim _{x_{i} \downarrow r}\left\{h_{\Psi_{\eta}, r}^{i}(s, x) p_{X, s}(x)\right\}$ exists and is finite a.e. over $\mathbb{R}_{+}^{n-1}$, where $\Psi_{\eta}$ is defined in the same way as in Lemma 2 . Then, we have

$$
\mathrm{E}\left[\int_{0}^{t} \Psi\left(X_{s}\right) L^{i}(\mathrm{~d} s, r)\right]=\int_{0}^{t} \int_{\mathbb{R}_{+}^{n-1}} \lim _{x_{i} \downarrow r}\left\{h_{\Psi_{\eta}, r}^{i}(s, x) p_{X, s}(x)\right\} \mathrm{d} x_{\neq i} \mathrm{~d} s .
$$

Proof. Follows from Lemma 2 by the same arguments as in the proof of Corollary 2.1 of [11].

Lemma 3. Let $t \in \mathbb{R}_{+}$, and let $i \in K \subseteq\{1, \ldots, n\}$. Assume that there exists $j \in K, j \neq i$, such that $\mathrm{P}\left\{\omega \in \Omega: a_{j, j}(\omega, s, x)>0\right.$ for all $\left.x \in \bigcap_{k \in K \backslash\{i\}} \partial_{k}\right\}=1$ for a.e. $s \in[0, t]$. Then, we have

$$
\int_{0}^{t} \mathbf{1}\left\{X_{s} \in \bigcap_{k \in K} \partial_{k}\right\} L^{i}(\mathrm{~d} s, 0)=0 \text { a.s. }
$$

Proof. From Lemma 1 we conclude that $P_{s}^{X}\left(\bigcap_{k \in K \backslash\{i\}} \partial_{k}\right)=0$ for a.e. $s \in[0, t]$. Thus, with $\Phi(\omega, x)=1$ and $\Psi(\omega, x)=\Psi(x)=\mathbf{1}\left\{x \in \bigcap_{k \in K} \partial_{k}\right\},(\omega, x) \in \Omega \times \mathbb{R}_{+}^{n}$, for each $\eta \in$ $(0, \infty)$, we have

$$
\begin{aligned}
0 & =\int_{0}^{t} \int_{\mathbb{R}_{+}^{n}(i, 0, \eta)} \mathbf{1}\left\{x \in \bigcap_{k \in K \backslash\{i\}} \partial_{k}\right\} h_{\Phi, 0}^{i}(s, x) P_{s}^{X}(\mathrm{~d} x) \mathrm{d} s \\
& =\int_{0}^{t} \int_{\mathbb{R}_{+}^{n}(i, 0, \eta)} h_{\Psi, 0}^{i}(s, x) P_{s}^{X}(\mathrm{~d} x) \mathrm{d} s .
\end{aligned}
$$

The lemma now follows from Lemma 2 since $\int_{0}^{t} \mathbf{1}\left\{X_{s} \in \bigcap_{k \in K} \partial_{k}\right\} L^{i}(\mathrm{~d} s, 0) \geq 0$.

Using the previous lemmas, we can now state the main results of this section in Theorems 1 and 2 , below. 
Theorem 1. Let $t \in \mathbb{R}_{+}$, and assume that there exist $i, j \in K \subseteq\{1, \ldots, n\}, i \neq j$, such that

$$
\mathrm{P}\left\{\omega \in \Omega: a_{i, i}(\omega, s, x)>0 \text { for all } x \in \bigcap_{k \in K \backslash\{j\}} \partial_{k}\right\}=1
$$

and

$$
\mathrm{P}\left\{\omega \in \Omega: a_{j, j}(\omega, s, x)>0 \text { for all } x \in \bigcap_{k \in K \backslash\{i\}} \partial_{k}\right\}=1
$$

for a.e. $s \in[0, t]$. Then, for each $q \in K$, we have

$$
\int_{0}^{t} \mathbf{1}\left\{X_{s} \in \bigcap_{k \in K} \partial_{k}\right\} \mathrm{d} Z_{s}^{q}=0 \text { a.s. }
$$

Proof. For $k \in\{1, \ldots, n\}$, write, analogously for $X_{s}^{0_{k}}, X_{s-}^{0_{k}}$ for $X_{s-}$ when its $k$ th component $X_{s-}^{k}$ is replaced by 0 . This same notation is used for $U \subseteq\{1, \ldots, n\}$, i.e. $X_{s-}^{0_{U}}$ denotes $X_{s-}$ when all its components $X_{s-}^{k}$ with $k \in U$ are replaced by 0 . Moreover, recall that, for $D \in \mathbb{R}^{n \times n}$ and $U \subsetneq\{1, \ldots, n\}, D^{(U)}$ denotes the principal submatrix obtained from $D$ by deleting its $k$ th row and column for all $k \in U$. Define the $\left(\mathcal{F}_{S}\right)_{s \geq 0}$-adapted, $\mathbb{R}^{n \times n}$-valued process $Q$ as follows:

$$
Q_{s}^{k, l}:= \begin{cases}\mathbf{1}\left\{X_{s-} \in \partial_{k}\right\} R_{k, l}\left(s, X_{s-}^{0_{k}}\right) & \text { if } k \neq l, \\ R_{k, k}\left(s, X_{s-}^{0_{k}}\right) & \text { if } k=l,\end{cases}
$$

where $s \in \mathbb{R}_{+}$and $k, l \in\{1, \ldots, n\}$. Furthermore, define the random index set $\Lambda_{s}, s \in \mathbb{R}_{+}$, as $\left\{k \in\{1, \ldots, n\}: X_{s-} \notin \partial_{k}\right\}$, and let $\Lambda_{s}^{\mathrm{c}}$ be its complement with respect to $\{1, \ldots, n\}$. Then, it is easy to see that, for each $s \in \mathbb{R}_{+}$and $\omega \in \Omega$, we have

$$
\operatorname{det}\left[Q_{s}\right]= \begin{cases}\operatorname{det}[R(s, 0)] & \text { if } \Lambda_{s}=\varnothing, \\ \prod_{k=1}^{n} R_{k, k}\left(s, X_{s-}^{0_{k}}\right) & \text { if }\left|\Lambda_{s}\right|=n, \\ \operatorname{det}\left[\left(R\left(s, X_{s-}^{0_{\Lambda_{s}^{c}}}\right)\right)^{\left(\Lambda_{s}\right)}\right] \prod_{k \in \Lambda_{s}} R_{k, k}\left(s, X_{s-}^{0_{k}}\right) & \text { if } 0<\left|\Lambda_{s}\right|<n,\end{cases}
$$

where $\operatorname{det}[\cdot]$ denotes the determinant of the corresponding matrix in $\mathbb{R}^{n \times n}$, and where 0 in $R(s, 0)$ is of course $0 \in \mathbb{R}^{n}$. Therefore, since, by assumption, in the paper we have $\mathrm{P}\{\omega \in$ $\Omega: R(\omega, s, x) \in \mathcal{R}_{n}$ for all $\left.(s, x) \in \mathbb{R}_{+} \times \partial_{0}\right\}=1$, we conclude that $\operatorname{det}\left[Q_{s}\right]>0$ a.s. for all $s \in \mathbb{R}_{+}$. Then, setting $r_{s}:=Q_{s}^{-1}, s \in \mathbb{R}_{+}$, applying Meyer-Itô's formula [12, Theorem 51] to each $X^{k}$ with convex function $f\left(x_{k}\right)=\left(x_{k}\right)^{+}:=\max \left\{0, x_{k}\right\}, x_{k} \in \mathbb{R}$, and using (3) along with the fact that $X^{k} \geq 0$, by the same arguments as in [11, Lemma 2.5], we obtain, for each $k \in\{1, \ldots, n\}$,

$$
Z_{.}^{k}=\sum_{l=1}^{n} \int_{0}^{\cdot} r_{s}^{k, l} \mathbf{1}\left\{X_{s} \in \partial_{l}\right\}\left(\frac{1}{2} L^{l}(\mathrm{~d} s, 0)-b^{l}\left(s, X_{s}\right) \mathrm{d} s\right) \quad \text { a.s. }
$$

The proof of the theorem now follows from Lemmas 1 and 3 by the same arguments as in the proof of Theorem 3.1 of [11]. 
Theorem 2. Assume that, for each $i \in\{1, \ldots, n\}$, we have, for a.e. $t \in \mathbb{R}_{+}, \mathrm{P}\{\omega \in \Omega$ : $a_{i, i}(\omega, t, x)>0$ for all $\left.x \in \partial_{i}\right\}=1$. Then, for each $i \in\{1, \ldots, n\}$, we have

$$
Z^{i}=\frac{1}{2} \int_{0}^{.} \frac{L^{i}(\mathrm{~d} s, 0)}{R_{i, i}\left(s, X_{s}\right)} \quad \text { a.s. }
$$

Proof. Using (4), Lemmas 1 and 3, and noting that $r_{s}^{i, i}=1 / R_{i, i}\left(s, X_{s-}\right)$ on $\{(\omega, s) \in$ $\left.\Omega \times \mathbb{R}_{+}: X_{s-}(\omega) \in \partial_{i}^{\circ}\right\}$, by the same arguments as in the proof of Theorem 3.2 of [11] we conclude that, for each $i \in\{1, \ldots, n\}$,

$$
Z_{t}^{i}=\frac{1}{2} \int_{0}^{t} \frac{L^{i}(\mathrm{~d} s, 0)}{R_{i, i}\left(s, X_{s}\right)} \quad \text { a.s. }
$$

for each $t \in \mathbb{R}_{+}$. Indistinguishability follows from a.s. sample path continuity.

Corollary 2. Assume the same as in Theorem 2 above. Then, for each $i \in\{1, \ldots, n\}$, the random measures (in $t) \mathrm{d} Z_{t}^{i}$ and $L^{i}(\mathrm{~d} t, 0)$ are a.s. supported by the same set in $\mathbb{R}_{+}$and, moreover, this set is contained in $\left\{t \in \mathbb{R}_{+}: X_{t} \in \partial_{i}^{\circ}\right\}$.

Proof. Follows directly from Lemma 3 (or Theorem 1) and Theorem 2.

Corollary 3. For each $i \in\{1, \ldots, n\}$, let $V^{i}:=X^{i}-Y^{i}$ with $Y_{.}^{i}:=\int_{0}^{\cdot} R_{i, i}\left(s, X_{s-}\right) \mathrm{d} Z_{s}^{i}=$ $\int_{0}^{i} R_{i, i}\left(s, X_{s}\right) \mathrm{d} Z_{s}^{i}$. Then, under the same assumptions as in Theorem 2 above, for each $i \in$ $\{1, \ldots, n\}$, we have

$$
L^{i}(\cdot, 0)=2 \sup _{s \in[0, \cdot]} \max \left\{-V_{s}^{i}, 0\right\} \text { a.s. }
$$

Proof. Let $i \in\{1, \ldots, n\}$. Since, by definition, $X^{i}=V^{i}+Y^{i}$, and $Y^{i}$ is clearly continuous, null at zero, nondecreasing $\left(\mathrm{P}\left\{\omega \in \Omega: R_{i, i}(\omega, t, x)>0\right.\right.$ for all $\left.\left.(t, x) \in \mathbb{R}_{+} \times \partial_{i}\right\}=1\right)$, and such that $\int_{\mathbb{R}_{+}} X_{s}^{i} \mathrm{~d} Y_{s}^{i}=0$, we conclude (see, for example, [3], [17], and [18]) that $Y_{\text {. }}^{i}=$ $\sup _{s \in[0, \cdot]} \max \left\{-V_{s}^{i}, 0\right\}$. (Note that, since $X$ is constrained to lie in $\mathbb{R}_{+}^{n}$, we have $X_{0} \geq 0$ and $\Delta X_{t} \geq-X_{t-}$ for all $t>0$, and, therefore, $\sup _{s \in[0, \cdot]} \max \left\{-V_{s}^{i}, 0\right\}$ is continuous, in agreement with this last equality and the definition of $Y^{i}$.) Therefore,

$$
\int_{0} R_{i, i}\left(s, X_{s}\right) \mathrm{d} Z_{s}^{i}=\sup _{s \in[0, \cdot]} \max \left\{-V_{s}^{i}, 0\right\} \quad \text { a.s. }
$$

The corollary now follows from Theorem 2 .

Remark 3. The boundary behavior characterization provided in this section can naturally be carried over to the case of a hyper-rectangular state space, as it was done in [11] for the simpler reflected diffusion model considered there. As the reader can easily verify, all the results in this section also extend to the case of a wedge in $\mathbb{R}_{+}^{n}$ as the state space.

\section{Necessary and sufficient conditions for a product-form distribution in the stationary regime}

In this section we extend the conditions given in [10] for a product-form distribution in the stationary regime, assuming that this regime and a corresponding density with respect to the Lebesgue measure exist, to the case when the drift and diffusion coefficients are allowed to be random fields over space, and when the reflection matrix is an $\mathbb{R}^{n \times n}$-valued random field over space as well. (As the reader may furthermore notice, some of the assumptions made 
in [10] will now be replaced by less restrictive assumptions.) Specifically, the model is the same as in Section 2, (3), but of course with $b, \gamma$, and $R$ being time independent, and with an appropriate structure for the jumps (described below). To make the extension to this setting clear, we first consider the case of nonrandom $b, \gamma$, and $R$ (i.e. when they do not depend on $\omega \in \Omega$ ), giving some explicit computational examples in Subsection 4.1. The fully random case is considered in Subsection 4.2. Similarly as in Section 3, the proofs given here focus on the points of difference between the corresponding proofs given in [10].

Consider the model in (3), but for the case when $b, \gamma$, and $R$ do not depend on time or $\omega \in \Omega$, i.e.

$$
\mathrm{d} X_{t}=b\left(X_{t-}\right) \mathrm{d} t+\gamma\left(X_{t-}\right) \mathrm{d} W_{t}+\Delta X_{t}+R\left(X_{t-}\right) \mathrm{d} Z_{t} .
$$

Aside from the time and $\omega$ independence, everything is as in Section 2, but we now make the following additional assumptions for each $i, j \in\{1, \ldots, n\}$.

- $b_{i}(x)$ and $a_{i, j}(x)$ depend only on $x \in \mathbb{R}_{+}^{n}$ through their $i$ th coordinate and $i, j$ th coordinates, respectively, i.e. $b_{i}(x)=b_{i}\left(x_{i}\right)$ and $a_{i, j}(x)=a_{i, j}\left(x_{i}, x_{j}\right)$. Note that the diffusion coefficients $a_{i, j}$ satisfy this $x$-dependence condition when, for example, $\gamma_{i, j}(x)=\gamma_{i, j}\left(x_{i}\right)$. Moreover, we assume that $a_{i, i}(0)>0$ and that $a_{i, i}(0+)$ exists and is finite.

- $R_{i, j}(x)$ depends only on $x \in \mathbb{R}_{+}^{n}$ through its $i$ th coordinate, i.e. $R_{i, j}(x)=R_{i, j}\left(x_{i}\right)$. Note that, since the direction of reflection upon hitting $\partial_{j}^{\circ}$ is given by the $j$ th column of $R$, $R_{j}(x):=\left(R_{1, j}\left(x_{1}\right), \ldots, R_{j, j}(0), \ldots, R_{n, j}\left(x_{n}\right)\right)$, this condition on $R$ is not completely restrictive in the sense that $R_{j}(x)$ is still allowed to depend on the position over that face. Also, note that, since $R_{j}(\cdot)$ only comes into play over $\partial_{j}$, it is equivalent to assuming an $x$-dependence of the form $R_{i, j}(x)=R_{i, j}\left(x_{i}, x_{j}\right)$. Moreover, we assume that, for each $i \neq j$, there exists a constant $C_{i, j} \in \mathbb{R}_{+}$such that $\left|R_{i, j}\left(x_{i}\right)\right| \leq C_{i, j}$ for all $x_{i} \in \mathbb{R}_{+}$.

Also, we consider the following structure for the jumps in $X$. Here $\mathbb{N}_{0}:=\{0,1,2, \ldots\}$.

- $\sum_{0<s \leq .} \Delta X_{s}(\omega)=\int_{0} \int_{\mathbb{R}^{n}} z \Pi(\omega, \mathrm{d} s, \mathrm{~d} z)$, where $\Pi$ is an $\mathbb{N}_{0}$-valued random measure over $\mathbb{R}_{+} \times \mathbb{R}^{n}$ with $\int_{0} \int_{\mathbb{R}^{n}} z \Pi(\omega, \mathrm{d} s, \mathrm{~d} z)\left(\mathcal{F}_{t}\right)_{t \geq 0}$-adapted, $\Pi\left(\omega,\{s\}, \mathbb{R}^{n}\right) \in\{0,1\}$ for each $(\omega, s) \in \Omega \times \mathbb{R}_{+}$, and $\int_{0}^{0} \int_{x_{i=1}^{n}\left(-\infty,-X_{s-}^{i}(\omega)\right)} z \Pi(\omega, \mathrm{d} s, \mathrm{~d} z) \equiv 0$ for each $\omega \in \Omega$. In particular, we assume that $\Pi$ admits a predictable compensator of the form $\lambda(\omega) K(\omega, s, \mathrm{~d} z) \mathrm{d} s$, where

1. $K(\omega, s, \mathrm{~d} z)$ is a Markovian, predictable transition kernel of $\left(\Omega \times \mathbb{R}_{+}, \mathcal{P}\right)$ into $\left(\mathbb{R}^{n}, \mathcal{B}\left(\mathbb{R}^{n}\right)\right.$ ), where $\mathcal{P}$ denotes the corresponding predictable $\sigma$-field on $\Omega \times \mathbb{R}_{+}$, taking the form $K\left(X_{s-}(\omega), \mathrm{d} z\right)$ with $K(x, \mathrm{~d} z)$ a probability transition kernel of $\left(\mathbb{R}_{+}^{n}, \mathcal{B}\left(\mathbb{R}_{+}^{n}\right)\right)$ into $\left(\mathbb{R}^{n}, \mathcal{B}\left(\mathbb{R}^{n}\right)\right)$ of the form $\prod_{i=1}^{n} K_{i}\left(x_{i}, \mathrm{~d} z_{i}\right)$, where each one-dimensional marginal distribution $K_{i}\left(x_{i}, \cdot\right)$ is such that $K_{i}\left(x_{i},\{0\}\right)=$ $K_{i}\left(x_{i},\left(-\infty,-x_{i}\right)\right)=0$ for each $x_{i} \geq 0$; and,

2. the intensity $\lambda$ is independent of $\left(X_{t-}\right)_{t \geq 0}$ and of the form $\sum_{i=1}^{n} \lambda_{i}$, where $\lambda_{i}(\omega) \in$ $[0, \infty), \omega \in \Omega$, is the jump intensity associated with $X^{i}$ and the $\lambda_{i}$ s are independent.

Finally, we set $\bar{\lambda}_{i}:=\int_{\mathbb{R}_{+}} r \phi_{i}(\mathrm{~d} r)$, where $\phi_{i}(\mathrm{~d} r)$ is the distribution of $\lambda_{i}$.

We assume the existence of a unique-in-law weak solution to (5). Furthermore, we assume that the corresponding law $P_{t}^{X}$ is time invariant (or stationary), i.e. it does not depend on $t$, denoted as $P^{X}$, and that such a law admits a density with respect to the Lebesgue measure, 
denoted as $p_{X}(x), x \in \mathbb{R}_{+}^{n}$. To ease notation, from now on we will omit the $X$ in $P^{X}$ and $p_{X}$. In addition, we assume that the density $p$ and its one- and two-dimensional marginals, denoted respectively as $\left\{p_{i}\right\}_{i=1}^{n}$ and $\left\{p_{i, j}\right\}_{i, j=1}^{n}$ (note that, for convenience, we have also introduced $p_{i, i}$, identified of course with $p_{i}$ ), are such that, for each $i, j \in\{1, \ldots, n\}$, the following five assumptions are satisfied.

(A1) $\bar{\lambda}_{i} \int_{\mathbb{R}_{+}} \int_{-x_{i}}^{\infty}\left|z_{i}\right| K_{i}\left(x_{i}, \mathrm{~d} z_{i}\right) p_{i}\left(x_{i}\right) \mathrm{d} x_{i}<\infty$.

(A2) $\int_{\mathbb{R}_{+}}\left|b_{i}\left(x_{i}\right)\right| p_{i}\left(x_{i}\right) \mathrm{d} x_{i}<\infty$.

(A3) $\int_{\mathbb{R}_{+}^{2}}\left|a_{i, j}\left(x_{i}, x_{j}\right)\right| p_{i, j}\left(x_{i}, x_{j}\right) \mathrm{d} x_{i} \mathrm{~d} x_{j}<\infty$.

(A4) $p\left(0_{i}+\right)$ exists and is finite a.e. over $\mathbb{R}_{+}^{n-1}$.

(A5) There exists $\eta_{i}>0$ such that $\int_{\mathbb{R}_{+}^{n-1}} \sup _{x_{i} \leq \eta_{i}}\left\{a_{i, i}\left(x_{i}\right) p(x)\right\} \mathrm{d} x_{\neq i}<\infty$.

Note that when $p$ is in product form, i.e. when

$$
p(x)=\prod_{i=1}^{n} p_{i}\left(x_{i}\right) \quad \text { for a.e. } x \in \mathbb{R}_{+}^{n},
$$

assumptions (A4) and (A5) together become equivalent to the existence and finiteness of the limit $p_{i}(0+)$ for each one-dimensional marginal $p_{i}$ (recall that $a_{i, i}(0+)$ is assumed to exist and to be finite for each $i$ ).

We define the operators $\mathcal{A}$ over $\mathcal{C}_{b}^{2}\left(\mathbb{R}_{+}^{n}\right)$ and $\mathcal{A}_{i}$ over $\mathcal{C}_{b}^{2}\left(\mathbb{R}_{+}\right), i \in\{1, \ldots, n\}$, as

$$
\begin{gathered}
{[\mathcal{A} f](x):=\sum_{i=1}^{n} b_{i}\left(x_{i}\right) \frac{\partial f}{\partial x_{i}}(x)+\frac{1}{2} \sum_{i, j=1}^{n} a_{i, j}\left(x_{i}, x_{j}\right) \frac{\partial^{2} f}{\partial x_{i} \partial x_{j}}(x),} \\
{\left[\mathcal{A}_{i} f\right]\left(x_{i}\right):=b_{i}\left(x_{i}\right) f^{\prime}\left(x_{i}\right)+\frac{1}{2} a_{i, i}\left(x_{i}\right) f^{\prime \prime}\left(x_{i}\right) .}
\end{gathered}
$$

Now we state some preliminary results towards the main result of this section, Theorem 3 .

Lemma 4. The stationary density $p$ satisfies the following equation for each $f \in \mathcal{C}_{b}^{2}\left(\mathbb{R}_{+}^{n}\right)$ :

$$
\begin{aligned}
0= & \int_{\mathbb{R}_{+}^{n}}[\mathcal{A} f](x) p(x) \mathrm{d} x+\frac{1}{2} \sum_{\substack{i, j=1 \\
i \neq j}}^{n} \frac{a_{j, j}(0+)}{R_{j, j}(0)} \int_{\mathbb{R}_{+}^{n-1}} \frac{\partial f}{\partial x_{i}}\left(0_{j}\right) R_{i, j}\left(x_{i}\right) p\left(0_{j}+\right) \mathrm{d} x_{\neq j} \\
& +\frac{1}{2} \sum_{i=1}^{n} a_{i, i}(0+) \int_{\mathbb{R}_{+}^{n-1}} \frac{\partial f}{\partial x_{i}}\left(0_{i}\right) p\left(0_{i}+\right) \mathrm{d} x_{\neq i} \\
& +\sum_{i=1}^{n} \bar{\lambda}_{i} \int_{\mathbb{R}_{+}^{n}} \int_{-x_{i}}^{\infty}\left(f\left(x+z_{i}\right)-f(x)\right) K_{i}\left(x_{i}, \mathrm{~d} z_{i}\right) p(x) \mathrm{d} x,
\end{aligned}
$$

where $x+z_{i}$ stands for $x+\hat{e}_{i} z_{i}$ with $\hat{e}_{i}$ denoting the usual ith unitary vector in $\mathbb{R}^{n}$. 
Proof. Let $f \in \mathcal{C}_{b}^{2}\left(\mathbb{R}_{+}^{n}\right)$. By applying Itô's formula [12, Theorem 33] to $X$ with $f$ and using (5), we obtain

$$
\begin{aligned}
f\left(X_{t}\right)-f\left(X_{0}\right)= & \sum_{i=1}^{n} \int_{0}^{t} \frac{\partial f}{\partial x_{i}}\left(X_{s-}\right) b_{i}\left(X_{s-}^{i}\right) \mathrm{d} s+\sum_{i, j=1}^{n} \int_{0}^{t} \frac{\partial f}{\partial x_{i}}\left(X_{s-}\right) \gamma_{i, j}\left(X_{s-}\right) \mathrm{d} W_{s}^{j} \\
& +\frac{1}{2} \sum_{i, j=1}^{n} \int_{0}^{t} \frac{\partial^{2} f}{\partial x_{i} \partial x_{j}}\left(X_{s-}\right) a_{i, j}\left(X_{s-}^{i}, X_{s-}^{j}\right) \mathrm{d} s+\sum_{0<s \leq t} \triangle f\left(X_{s}\right) \\
& +\sum_{i, j=1}^{n} \int_{0}^{t} \frac{\partial f}{\partial x_{i}}\left(X_{s-}\right) R_{i, j}\left(X_{s-}^{i}\right) \mathrm{d} Z_{s}^{j},
\end{aligned}
$$

where $\triangle f\left(X_{s}\right):=f\left(X_{s}\right)-f\left(X_{s-}\right)$. Now, since $a_{i, i}(0)>0$ for each $i$, from Theorem 2 , the last sum in (7) equals

$$
\frac{1}{2} \sum_{\substack{i, j=1 \\ i \neq j}}^{n} \frac{1}{R_{j, j}(0)} \int_{0}^{t} \frac{\partial f}{\partial x_{i}}\left(X_{s}\right) R_{i, j}\left(X_{s}^{i}\right) L^{j}(\mathrm{~d} s, 0)+\frac{1}{2} \sum_{i=1}^{n} \int_{0}^{t} \frac{\partial f}{\partial x_{i}}\left(X_{s}\right) L^{i}(\mathrm{~d} s, 0),
$$

where in the left-hand sum we have used the fact that, a.s., $L^{j}(s, 0)$ can increase only at times $s$ when $X_{s}^{j}=0$ (see Corollary 2 or [12, Theorem 50]). In addition, from assumptions (A4) and (A5), and the facts that $f \in \mathcal{C}_{b}^{2}\left(\mathbb{R}_{+}^{n}\right)$ (in particular, its first-order partial derivatives are bounded and continuous), that the $R_{i, j} s, i \neq j$, are bounded, and that the limits, $a_{i, i}(0+), i=1, \ldots, n$, exist and are finite, by using Corollary 1 we conclude that

$$
\mathrm{E}\left[\int_{0}^{t} \frac{\partial f}{\partial x_{i}}\left(X_{s}\right) R_{i, j}\left(X_{s}^{i}\right) L^{j}(\mathrm{~d} s, 0)\right]=t a_{j, j}(0+) \int_{\mathbb{R}_{+}^{n-1}} \frac{\partial f}{\partial x_{i}}\left(0_{j}\right) R_{i, j}\left(x_{i}\right) p\left(0_{j}+\right) \mathrm{d} x_{\neq j}
$$

for $i \neq j$ and

$$
\mathrm{E}\left[\int_{0}^{t} \frac{\partial f}{\partial x_{i}}\left(X_{s}\right) L^{i}(\mathrm{~d} s, 0)\right]=t a_{i, i}(0+) \int_{\mathbb{R}_{+}^{n-1}} \frac{\partial f}{\partial x_{i}}\left(0_{i}\right) p\left(0_{i}+\right) \mathrm{d} x_{\neq i} .
$$

Furthermore, since the jumps in different components of $X$ are independent, we have

$$
\mathrm{E}\left[\sum_{0<s \leq t} \Delta f\left(X_{S}\right)\right]=t \sum_{i=1}^{n} \bar{\lambda}_{i} \int_{\mathbb{R}_{+}^{n}} \int_{-x_{i}}^{\infty}\left(f\left(x+z_{i}\right)-f(x)\right) K_{i}\left(x_{i}, \mathrm{~d} z_{i}\right) p(x) \mathrm{d} x .
$$

All the remaining terms in (7) are handled the same way under expectation as in the proof of Lemma 1 of [10]. Dividing by $t>0$ completes the proof.

Corollary 4. Assume that $p$ is in product form, i.e. that $p(x)=\prod_{i=1}^{n} p_{i}\left(x_{i}\right)$ for a.e. $x \in \mathbb{R}_{+}^{n}$. Then, each one-dimensional marginal $p_{i}$ satisfies the following equation for each $f \in \mathcal{C}_{b}^{2}\left(\mathbb{R}_{+}\right)$:

$$
\begin{aligned}
0= & \int_{\mathbb{R}_{+}}\left[\mathcal{A}_{i} f\right]\left(x_{i}\right) p_{i}\left(x_{i}\right) \mathrm{d} x_{i}+\frac{1}{2} \sum_{\substack{j=1 \\
j \neq i}}^{n} \frac{a_{j, j}(0+) p_{j}(0+)}{R_{j, j}(0)} \int_{\mathbb{R}_{+}} f^{\prime}\left(x_{i}\right) R_{i, j}\left(x_{i}\right) p_{i}\left(x_{i}\right) \mathrm{d} x_{i} \\
& +\bar{\lambda}_{i} \int_{\mathbb{R}_{+}} \int_{-x_{i}}^{\infty}\left(f\left(x_{i}+z_{i}\right)-f\left(x_{i}\right)\right) K_{i}\left(x_{i}, \mathrm{~d} z_{i}\right) p_{i}\left(x_{i}\right) \mathrm{d} x_{i} \\
& +\frac{1}{2} f^{\prime}(0) a_{i, i}(0+) p_{i}(0+) .
\end{aligned}
$$


Proof. The proof follows straightforwardly from Lemma 4.

Remark 4. Note that if $p$ is in product form, by applying Meyer-Itô's formula [12, Theorem 51] to each $X^{i}$ with convex function $f\left(x_{i}\right)=\left(x_{i}\right)^{+}:=\max \left\{0, x_{i}\right\}, x_{i} \in \mathbb{R}$, using (5), taking expectations, and using Corollary 1 , we obtain, for each $i \in\{1, \ldots, n\}$,

$$
-\frac{1}{2} \sum_{\substack{j=1 \\ j \neq i}}^{n} \frac{a_{j, j}(0+) p_{j}(0+)}{R_{j, j}(0)} \int_{\mathbb{R}_{+}} R_{i, j}\left(x_{i}\right) p_{i}\left(x_{i}\right) \mathrm{d} x_{i}-\frac{1}{2} a_{i, i}(0+) p_{i}(0+)=\Lambda_{i},
$$

where

$$
\Lambda_{i}:=\int_{\mathbb{R}_{+}} b_{i}\left(x_{i}\right) p_{i}\left(x_{i}\right) \mathrm{d} x_{i}+\bar{\lambda}_{i} \int_{\mathbb{R}_{+}} \int_{-x_{i}}^{\infty} z_{i} K_{i}\left(x_{i}, \mathrm{~d} z_{i}\right) p_{i}\left(x_{i}\right) \mathrm{d} x_{i} .
$$

Then, in the case when the reflection matrix $R$ is constant, by using the above expression along with (8) we obtain, for each marginal $p_{i}$, an equation involving only its own limit at the boundary $p_{i}(0+)$, instead of the whole set $\left\{p_{j}(0+)\right\}_{j=1}^{n}$ as in (8) (see [10, Corollaries 2 and 3, and Lemma 2]). Unfortunately, this is not the case when $R$ is nonconstant, as we are considering here. From a computational point of view, this makes the problem much harder to work out, as we will see in Subsection 4.1. In the same way, it is now not possible to derive a condition, in terms of the net drifts (including reflections) in each dimension, ensuring that $p_{i}(0+)>0$ for each marginal $p_{i}$, in contrast as well to the case of a constant reflection matrix $R$; see [10, Remark 3].

Remark 5. Similar to [10, Remarks 2 and 4], we note that, when $p$ is product form, Lemma 4 and Corollary 4 respectively uniquely characterize $p$ and each one-dimensional marginal $p_{i}$ in an a.e. sense. Note this is also the case if (6) is satisfied for all $f(x)=\prod_{i=1}^{n} f_{i}\left(x_{i}\right)$ with $f_{i} \in$ $\mathcal{C}_{b}^{2}\left(\mathbb{R}_{+}\right)$for each $i$, or, equivalently, if we consider (6) along with the family $\left\{\mathrm{e}^{-<\alpha, \cdot>}\right\}_{\alpha \in \mathbb{R}_{+}^{n}} \subset$ $\mathcal{C}_{b}^{2}\left(\mathbb{R}_{+}^{n}\right)$.

Lemma 5, below, though not used in Theorem 3, below, provides a useful alternative expression to (8). A simple example of its use will be considered in Subsection 4.1.

Lemma 5. Assume that, for each $i, j \in\{1, \ldots, n\}, i \neq j, R_{i, j}, b_{i}, a_{i, i}$, and $p_{i} \in \mathcal{C}\left(\mathbb{R}_{+}\right)$, $a_{i, i} p_{i} \in \mathcal{C}^{1}\left(\mathbb{R}_{+}\right)$such that the Laplace transform of $\left(a_{i, i} p_{i}\right)^{\prime}$ exists and $K_{i}\left(x_{i}, \cdot\right)$ is absolutely continuous with respect to the Lebesgue measure for each $x_{i} \in \mathbb{R}_{+}$with density $k_{i}\left(x_{i}, z_{i}\right)$, i.e. $K_{i}\left(x_{i}, \mathrm{~d} z_{i}\right)=k_{i}\left(x_{i}, z_{i}\right) \mathrm{d} z_{i}$, where, of course, $k_{i}\left(x_{i}, z_{i}\right) \equiv 0$ for $z_{i} \in\left(-\infty,-x_{i}\right)$. Then, each $p_{i}$ satisfies (8) for all $f \in \mathcal{C}_{b}^{2}\left(\mathbb{R}_{+}\right)$if and only if it satisfies, for all $x_{i} \in \mathbb{R}_{+}$,

$$
\begin{aligned}
0= & \frac{1}{2}\left(a_{i, i} p_{i}\right)^{\prime}\left(x_{i}\right)-p_{i}\left(x_{i}\right)\left(b_{i}\left(x_{i}\right)-\frac{1}{2} \sum_{\substack{j=1 \\
j \neq i}}^{n} \frac{a_{j, j}(0) p_{j}(0)}{R_{j, j}(0)} R_{i, j}\left(x_{i}\right)\right) \\
& +\bar{\lambda}_{i}\left(\int_{0}^{x_{i}} \int_{0}^{\infty} k_{i}(\varsigma, u-\varsigma) p_{i}(\varsigma) \mathrm{d} \varsigma \mathrm{d} u-\int_{0}^{x_{i}} p_{i}(\varsigma) \mathrm{d} \varsigma\right) .
\end{aligned}
$$

Proof. As in [10, Lemma 3], the necessity follows by considering $f\left(x_{i}\right)=\exp \left(-\alpha x_{i}\right)$ with $\alpha>0$ in (8), and then taking the inverse Laplace transform. Similarly, the sufficiency follows by considering $f \in \mathcal{C}_{b}^{2}\left(\mathbb{R}_{+}\right)$, multiplying (10) by $-f^{\prime}\left(x_{i}\right)$, and then integrating over $\mathbb{R}_{+}$using the fact that, by assumption (A3) with $i=j, a_{i, i}\left(x_{i}\right) p_{i}\left(x_{i}\right) \rightarrow 0$ as $x_{i} \uparrow \infty$. 
Now we state the main result of this section.

Theorem 3. The stationary density $p$ is in product form if and only if, for each $i, j \in\{1, \ldots, n\}$, $i \neq j$, we have

$$
\begin{aligned}
a_{i, j}\left(x_{i}, x_{j}\right)= & \frac{a_{i, i}(0+) p_{i}(0+) R_{j, i}\left(x_{j}\right)}{2 R_{i, i}(0) p_{i}\left(x_{i}\right)} \int_{x_{i}}^{\infty} p_{i}(\varsigma) \mathrm{d} \varsigma \\
& +\frac{a_{j, j}(0+) p_{j}(0+) R_{i, j}\left(x_{i}\right)}{2 R_{j, j}(0) p_{j}\left(x_{j}\right)} \int_{x_{j}}^{\infty} p_{j}(\varsigma) \mathrm{d} \varsigma
\end{aligned}
$$

for $P_{i, j}$-a.e. $\left(x_{i}, x_{j}\right) \in \mathbb{R}_{+}^{2}$, where $P_{i, j}$ denotes the joint (stationary) law of the tuple $\left(X_{t}^{i}, X_{t}^{j}\right)$ in $\mathbb{R}_{+}^{2}$.

Proof. For the necessity, from Lemma 4 and Corollary 4, we find, by the same arguments as in the proof of Theorem 1 of [10], that, for each $i, j \in\{1, \ldots, n\}, i \neq j$, and all $f_{i}, f_{j} \in \mathcal{C}_{b}^{2}\left(\mathbb{R}_{+}\right)$,

$$
\int_{\mathbb{R}_{+}^{2}} f_{i}^{\prime}\left(x_{i}\right) f_{j}^{\prime}\left(x_{j}\right) a_{i, j}\left(x_{i}, x_{j}\right) p_{i}\left(x_{i}\right) p_{j}\left(x_{j}\right) \mathrm{d} x_{i} \mathrm{~d} x_{j}=-\frac{\Upsilon_{i, j}\left(f_{i}, f_{j}\right)+\Upsilon_{j, i}\left(f_{j}, f_{i}\right)}{2},
$$

where

$$
\begin{aligned}
\Upsilon_{i, j}\left(f_{i}, f_{j}\right):= & \frac{a_{j, j}(0+) p_{j}(0+)}{R_{j, j}(0)} \\
& \times \int_{\mathbb{R}_{+}} f_{i}^{\prime}\left(x_{i}\right) R_{i, j}\left(x_{i}\right) p_{i}\left(x_{i}\right) \mathrm{d} x_{i}\left(f_{j}(0)-\int_{\mathbb{R}_{+}} f_{j}\left(x_{j}\right) p_{j}\left(x_{j}\right) \mathrm{d} x_{j}\right)
\end{aligned}
$$

and the corresponding symmetric expression for $\Upsilon_{j, i}\left(f_{j}, f_{i}\right)$. Then, by taking $f_{k}\left(x_{k}\right)=$ $\exp \left(-\alpha_{k} x_{k}\right)$ with $\alpha_{k}>0, k \in\{i, j\}$, we find that

$$
\begin{aligned}
\mathcal{L}_{2}\left[a_{i, j} p_{i} p_{j}\right]\left(\alpha_{i}, \alpha_{j}\right)= & \frac{a_{i, i}(0+) p_{i}(0+)}{2 R_{i, i}(0)} \mathcal{L}\left[R_{j, i} p_{j}\right]\left(\alpha_{j}\right)\left(\frac{1}{\alpha_{i}}-\frac{\mathcal{L}\left[p_{i}\right]\left(\alpha_{i}\right)}{\alpha_{i}}\right) \\
& +\frac{a_{j, j}(0+) p_{j}(0+)}{2 R_{j, j}(0)} \mathcal{L}\left[R_{i, j} p_{i}\right]\left(\alpha_{i}\right)\left(\frac{1}{\alpha_{j}}-\frac{\mathcal{L}\left[p_{j}\right]\left(\alpha_{j}\right)}{\alpha_{j}}\right)
\end{aligned}
$$

for all $\alpha_{i}, \alpha_{j}>0$, and, therefore, the necessity follows. The sufficiency follows from Lemma 4 and Remark 5, again by the same arguments as in the proof of Theorem 1 of [10]. This completes the proof.

\subsection{Some specific cases and their related equations}

Consider the case when, for each $i \in\{1, \ldots, n\}$, we have $\lambda_{i} \equiv 0$ (i.e. there are no jumps) and $a_{i, i}\left(x_{i}\right)>0$ for all $x_{i} \in \mathbb{R}_{+}$. Furthermore, assume that the hypotheses of Lemma 5 are satisfied. Then, from (10) we obtain, for each $i \in\{1, \ldots, n\}$ and all $x_{i} \in \mathbb{R}_{+}$,

$$
0=\left(a_{i, i} p_{i}\right)^{\prime}\left(x_{i}\right)-\left(2 b_{i}\left(x_{i}\right)+q_{i}\left(x_{i}\right)\right) p_{i}\left(x_{i}\right),
$$

where

$$
q_{i}\left(x_{i}\right):=\sum_{\substack{j=1 \\ j \neq i}}^{n} \frac{a_{j, j}(0) p_{j}(0)}{R_{j, j}(0)} R_{i, j}\left(x_{i}\right) .
$$


Therefore,

$$
p_{i}\left(x_{i}\right)=\frac{a_{i, i}(0) p_{i}(0)}{a_{i, i}\left(x_{i}\right)} \exp \left(\int_{0}^{x_{i}} \frac{2 b_{i}(\varsigma)+q_{i}(\varsigma)}{a_{i, i}(\varsigma)} \mathrm{d} \varsigma\right) .
$$

From the normalization condition, the boundary values, $\left\{p_{i}(0)\right\}_{i=1}^{n}$, satisfy the following equations:

$$
1=a_{i, i}(0) p_{i}(0) \int_{0}^{\infty} \frac{1}{a_{i, i}\left(x_{i}\right)} \exp \left(\int_{0}^{x_{i}} \frac{2 b_{i}(\varsigma)+q_{i}(\varsigma)}{a_{i, i}(\varsigma)} \mathrm{d} \varsigma\right) \mathrm{d} x_{i}, \quad i=1, \ldots, n .
$$

Equation (12) can be solved explicitly for the $p_{i}(0) \mathrm{s}$, from which the marginals themselves can then in turn be obtained via (11), when, for example, the reflection matrix is triangular. Indeed, consider, for instance, the case of lower triangular $R(\cdot)$, i.e. with $R_{i, j}\left(x_{i}\right) \equiv 0$ for $i, j \in\{1, \ldots, n\}, i<j$. Then, $q_{1}\left(x_{1}\right) \equiv 0$ and, therefore,

$$
p_{1}(0)=\left(a_{1,1}(0) \int_{0}^{\infty} \frac{1}{a_{1,1}\left(x_{1}\right)} \exp \left(2 \int_{0}^{x_{1}} \frac{b_{1}(\varsigma)}{a_{1,1}(\varsigma)} \mathrm{d} \varsigma\right) \mathrm{d} x_{1}\right)^{-1} .
$$

In a similar way, and proceeding in increasing order for $i$, we find that, for each $1<i \leq n$,

$$
\begin{aligned}
p_{i}(0)= & \left(a_{i, i}(0)\right. \\
& \left.\times \int_{0}^{\infty} \frac{1}{a_{i, i}\left(x_{i}\right)}\left(\int_{0}^{x_{i}} \frac{2 b_{i}(\varsigma)+\sum_{j=1}^{i-1}\left(a_{j, j}(0) p_{j}(0) / R_{j, j}(0)\right) R_{i, j}(\varsigma)}{a_{i, i}(\varsigma)} \mathrm{d} \varsigma\right) \mathrm{d} x_{i}\right)^{-1} .
\end{aligned}
$$

Of course, we require the model coefficients to be such that $p_{i}(0) \in(0, \infty), i \in\{1, \ldots, n\}$. In this case, if the stationary regime and a corresponding density $p$, satisfying all the conditions stated at the beginning of this section, exist and, furthermore, if the product-form condition of Theorem 3 is satisfied with the marginal densities given by (11) and the boundary values above, then, for a.e. $x \in \mathbb{R}_{+}^{n}$,

$$
\begin{aligned}
p(x)= & \prod_{i=1}^{n} p_{i}\left(x_{i}\right) \\
= & \frac{a_{1,1}(0) p_{1}(0)}{a_{1,1}\left(x_{1}\right)} \exp \left(2 \int_{0}^{x_{1}} \frac{b_{1}(\varsigma)}{a_{1,1}(\varsigma)} \mathrm{d} \varsigma\right) \\
& \times \prod_{i=2}^{n} \frac{a_{i, i}(0) p_{i}(0)}{a_{i, i}\left(x_{i}\right)} \exp \left(\int_{0}^{x_{i}} \frac{2 b_{i}(\varsigma)+\sum_{j=1}^{i-1}\left(a_{j, j}(0) p_{j}(0) / R_{j, j}(0)\right) R_{i, j}(\varsigma)}{a_{i, i}(\varsigma)} \mathrm{d} \varsigma\right)
\end{aligned}
$$

Explicit resolution of (12) for state dependent but nontriangular reflection matrix $R(\cdot)$ is in general not possible to carry out. It might result in an explicitly solvable system of equations however, in specific applications with given sets of model coefficients. In the same way, the $p_{i}(0)$ s can be computed explicitly in the simple case where the reflection matrix $R$ is constant, when a set of linear equations for the $p_{i}(0) \mathrm{s}$ can be derived instead from (9) (with $\bar{\lambda}_{i}=\lambda_{i} \equiv 0$ for each $i$ ) if in addition the drift $b$ is constant, or (12) can be used directly to solve for each $p_{i}(0)$ when $R$ is diagonal $\left(q_{i}\left(x_{i}\right) \equiv 0\right.$ for each $\left.i\right)$; see [10, Subsection 5.1].

On the other hand, the presence of jumps makes it impossible to derive an explicit solution for the nonconstant reflection matrix $R(\cdot)$. Indeed, consider, for example, the simple case 
when, for each $i \in\{1, \ldots, n\}$, we have $b_{i}\left(x_{i}\right)=b_{i}$ (a constant), $a_{i, i}\left(x_{i}\right)=a_{i, i}>0$ (a strictly positive constant), and $K_{i}\left(x_{i}, \mathrm{~d} z_{i}\right)=k_{i}\left(x_{i}, z_{i}\right) \mathrm{d} z_{i}=k_{i}\left(z_{i}\right) \mathrm{d} z_{i}$ with $k_{i}\left(z_{i}\right) \equiv 0$ for $z_{i}<0$. From Corollary 4 with $f\left(x_{i}\right)=\exp \left(-\alpha_{i} x_{i}\right), \alpha_{i}>0$, we obtain, for each $i \in\{1, \ldots, n\}$ and all $\alpha_{i}>0$,

$$
\begin{aligned}
0= & \mathcal{L}\left[p_{i}\right]\left(\alpha_{i}\right)\left(\frac{1}{2} \alpha_{i} a_{i, i}-b_{i}+\frac{\bar{\lambda}_{i} \mathcal{L}\left[k_{i}\right]\left(\alpha_{i}\right)-\bar{\lambda}_{i}}{\alpha_{i}}\right) \\
& -\frac{1}{2} a_{i, i} p_{i}(0+)-\frac{1}{2} \sum_{\substack{j=1 \\
j \neq i}}^{n} \frac{a_{j, j} p_{j}(0+)}{R_{j, j}(0)} \mathcal{L}\left[R_{i, j} p_{i}\right]\left(\alpha_{i}\right) .
\end{aligned}
$$

Then, even with lower triangular $R(\cdot)$ as before, though it is possible to solve for $\mathcal{L}\left[p_{1}\right](\cdot)$ in terms of $p_{1}(0+)$, from which in principle and via the inverse Laplace transform $p_{1}$ can be obtained and $p_{1}(0+)$ determined from the normalization condition, it is not possible to solve for $\mathcal{L}\left[p_{2}\right](\cdot)$, and similarly for higher indices $i$, owing to the additional term $\mathcal{L}\left[R_{2,1} p_{2}\right](\cdot)$.

All computations can be carried out explicitly however in the case of a not necessarily triangular but constant reflection matrix $R$, where the boundary values, $p_{i}(0+), i=1 \ldots, n$, are determined from an appropriate system of linear equations. For the details involved in this case, as well as for an example with exponentially distributed jumps, see [10, Subsection 5.2].

\subsection{Fully random case}

In this subsection we consider the case when $b, \gamma$, and $R$ are random fields over the space variable $x \in \mathbb{R}_{+}^{n}$. As we will see, all the results exposed so far in this section generalize to this case. The difficulty in this general setting arises from an applied or computational point of view. To check whether or not some of the assumptions to be made hold true, knowledge of the conditional statistics for the different model coefficients given $X$ is required. Hence, prior knowledge of joint statistics, as well as of the statistics of $X$, is in general needed, which supposes to some extent prior knowledge of whether or not $p$ factorizes as the product of its one-dimensional marginals. Therefore, the applicability of the results in this setting is limited to cases where, maybe because of the underlying dynamics or 'physics' involved, the model is set up by means of conditional statistics for its different coefficients.

Specifically, we now consider the same general model as in Section 2, (3), but of course with time independent $b, \gamma$, and $R$, i.e.

$$
\mathrm{d} X_{t}=b\left(\omega, X_{t-}\right) \mathrm{d} t+\gamma\left(\omega, X_{t-}\right) \mathrm{d} W_{t}+\Delta X_{t}+R\left(\omega, X_{t-}\right) \mathrm{d} Z_{t},
$$

where, aside from this time independence, everything is as in Section 2. Note that the adaptedness requirement on the coefficients of $b, \gamma$, and $R$ now reduces to $\mathcal{F}_{0} \otimes \mathscr{B}\left(\mathbb{R}_{+}^{n}\right)-\mathscr{B}(\mathbb{R})$ measurability. The structure for the jumps in $X$ is the same as the one detailed at the beginning of this section.

Of course, as we did before in this section, we assume the existence of a unique-in-law weak solution to (13), time invariance of the corresponding law of $X_{t}$, and that a respective density with respect to the Lebesgue measure, $p(x), x \in \mathbb{R}_{+}^{n}$, exists. Furthermore, we assume that all conditional expectations considered below (conditions for their well definiteness are also given below), such as $\mathrm{E}\left[b_{i}\left(X_{t}\right) \mid \sigma_{t}\right](\omega)$ (recall that $\sigma_{t}$ denotes the $\sigma$-algebra generated by $X_{t}$ ), do not depend explicitly on $t$ but just depend on $t$ through $X_{t}$. We continue to assume that the density $p$ satisfies assumption (A4) and that assumption (A1) is also satisfied by its one-dimensional marginals. In addition, we assume that, for each $i \in\{1, \ldots, n\}, \mathrm{P}\left\{\omega \in \Omega: a_{i, i}(\omega, x)>0\right.$ for 
all $\left.x \in \partial_{i}\right\}=1$, and that, for each $i, j \in\{1, \ldots, n\}, i \neq j$, there exists a constant $C_{i, j} \in \mathbb{R}_{+}$ such that

$$
\mathrm{P}\left\{\omega \in \Omega: \frac{\left|R_{i, j}(\omega, x)\right|}{R_{j, j}(\omega, x)} \leq C_{i, j} \text { for all } x \in \partial_{j}\right\}=1 .
$$

Also, assumptions (A2) and (A3) are now rephrased as follows. For each $i, j \in\{1, \ldots, n\}$,

(A2) $\mathrm{E}\left[\left|b_{i}\left(X_{t}\right)\right|\right]<\infty$,

(A3) $\mathrm{E}\left[\left|a_{i, j}\left(X_{t}\right)\right|\right]<\infty$.

From the assumptions above, it follows that, for each $i, j \in\{1, \ldots, n\}$, there exist $\hat{b}_{i}: \mathbb{R}_{+}^{n} \rightarrow$ $\mathbb{R}$ and $\hat{a}_{i, j}: \mathbb{R}_{+}^{n} \rightarrow \mathbb{R}$, Borel measurable, such that $\hat{b}_{i}\left(X_{t}(\omega)\right)=\mathrm{E}\left[b_{i}\left(X_{t}\right) \mid \sigma_{t}\right](\omega)$ a.s. and $\hat{a}_{i, j}\left(X_{t}(\omega)\right)=\mathrm{E}\left[a_{i, j}\left(X_{t}\right) \mid \sigma_{t}\right](\omega)$ a.s. (recall that all conditional expectations in this subsection are assumed to depend on $t$ only through $X_{t}$ ). Also, from the boundedness assumption on the ratios $R_{i, j}(x) / R_{j, j}(x)$ for $x \in \partial_{j}$ and the assumptions above, it follows that, for each $i, j \in\{1, \ldots, n\}, i \neq j$, there exists $h_{i, j}: \mathbb{R}_{+}^{n} \rightarrow \mathbb{R}$, Borel measurable, such that

$$
h_{i, j}\left(X_{t}(\omega)\right)=\mathrm{E}\left[\frac{R_{i, j}\left(X_{t}^{0_{j}}\right)}{R_{j, j}\left(X_{t}^{0_{j}}\right)} a_{j, j}\left(X_{t}\right) \mid \sigma_{t}\right](\omega) \text { a.s. }
$$

(recall that $X_{t}^{0_{j}}$ denotes $X_{t}$ when its $j$ th component $X_{t}^{j}$ is replaced by 0 ).

We assume that the functions $\hat{b}, \hat{a}$, and $h$, introduced in the previous paragraph, satisfy the following conditions for each $i, j \in\{1, \ldots, n\}$.

- $\hat{b}_{i}(x)$ and $\hat{a}_{i, j}(x)$ depend only on $x \in \mathbb{R}_{+}^{n}$ through their $i$ th coordinate and $i, j$ th coordinates, respectively, i.e. $\hat{b}_{i}(x)=\hat{b}_{i}\left(x_{i}\right)$ and $\hat{a}_{i, j}(x)=\hat{a}_{i, j}\left(x_{i}, x_{j}\right)$. Moreover, $\hat{a}_{i, i}(0+)$ exists and is finite.

- $h_{i, j}(x), i \neq j$, depends only on $x \in \mathbb{R}_{+}^{n}$ through its $i, j$ th coordinates, i.e. $h_{i, j}(x)=$ $h_{i, j}\left(x_{i}, x_{j}\right)$. Moreover, $h_{i, j}\left(x_{i}, 0+\right):=\lim _{x_{j} \downarrow 0} h_{i, j}\left(x_{i}, x_{j}\right)$ exists and is finite for each $x_{i} \in \mathbb{R}_{+}$.

Note that in the context discussed earlier regarding the applicability of the results in this general setting, the assumptions above on the $x$-dependence in $\hat{b}_{i}, \hat{a}_{i, j}$, and $h_{i, j}$ are rather hard to check a priori, involving stochastic independence between model coefficients and the corresponding component processes in $X$ with different indices. Also, note that, for the above $x$-dependencies to hold, it is not enough to extend the previous assumptions for the nonrandom case in the naive way, i.e. it is not enough to have $b_{i}\left(\omega, x_{i}\right), a_{i, j}\left(\omega, x_{i}, x_{j}\right)$, and $R_{i, j}\left(\omega, x_{i}\right)$.

Finally, assumption (A5) can now be rephrased as follows.

(A5) For each $i \in\{1, \ldots, n\}$, there exists $\eta_{i}>0$ such that

$$
\int_{\mathbb{R}_{+}^{n-1}} \sup _{x_{i} \leq \eta_{i}}\left\{\hat{a}_{i, i}\left(x_{i}\right) p(x)\right\} \mathrm{d} x_{\neq i}<\infty .
$$

Note that assumption (A5) together with the boundedness assumption on the ratios $R_{i, j}(x) /$ $R_{j, j}(x)$ for $x \in \partial_{j}$ imply that $\int_{\mathbb{R}_{+}^{n-1}} \sup _{x_{j} \leq \eta_{j}}\left\{\left|h_{i, j}\left(x_{i}, x_{j}\right)\right| p(x)\right\} \mathrm{d} x_{\neq j}<\infty$ for each $i, j \in$ $\{1, \ldots, n\}, i \neq j$.

We now state the corresponding generalizations of Lemma 4, Corollary 4, and Theorem 3 , respectively in Lemma 6, Corollary 5, and Theorem 4, below. The proofs are omitted since they follow 'mutatis-mutandis' by the same arguments as before. (Note that, as mentioned before in Lemma 2 in Section 3, the proof of Lemma 6 requires the use of Corollary 1 with random $\Psi_{\mathrm{s}}$.) 
Lemma 6. The stationary density $p$ satisfies the following equation for each $f \in \mathcal{C}_{b}^{2}\left(\mathbb{R}_{+}^{n}\right)$ :

$$
\begin{aligned}
0= & \int_{\mathbb{R}_{+}^{n}}[\hat{\mathcal{A}} f](x) p(x) \mathrm{d} x+\frac{1}{2} \sum_{\substack{i, j=1 \\
i \neq j}}^{n} \int_{\mathbb{R}_{+}^{n-1}} \frac{\partial f}{\partial x_{i}}\left(0_{j}\right) h_{i, j}\left(x_{i}, 0+\right) p\left(0_{j}+\right) \mathrm{d} x_{\neq j} \\
& +\frac{1}{2} \sum_{i=1}^{n} \hat{a}_{i, i}(0+) \int_{\mathbb{R}_{+}^{n-1}} \frac{\partial f}{\partial x_{i}}\left(0_{i}\right) p\left(0_{i}+\right) \mathrm{d} x_{\neq i} \\
& +\sum_{i=1}^{n} \bar{\lambda}_{i} \int_{\mathbb{R}_{+}^{n}} \int_{-x_{i}}^{\infty}\left(f\left(x+z_{i}\right)-f(x)\right) K_{i}\left(x_{i}, \mathrm{~d} z_{i}\right) p(x) \mathrm{d} x,
\end{aligned}
$$

where the same notation $x+z_{i}$ as in Lemma 4 is used, and where the operator $\hat{\mathcal{A}}$ is defined the same as $\mathcal{A}$ but with each $b_{i}$ and $a_{i, j}$ replaced correspondingly by $\hat{b}_{i}$ and $\hat{a}_{i, j}$.

Corollary 5. Assume that $p$ is in product form. Then, each one-dimensional marginal $p_{i}$ satisfies the following equation for each $f \in \mathcal{C}_{b}^{2}\left(\mathbb{R}_{+}\right)$:

$$
\begin{aligned}
0= & \int_{\mathbb{R}_{+}}\left[\hat{\mathcal{A}}_{i} f\right]\left(x_{i}\right) p_{i}\left(x_{i}\right) \mathrm{d} x_{i}+\frac{1}{2} \sum_{\substack{j=1 \\
j \neq i}}^{n} p_{j}(0+) \int_{\mathbb{R}_{+}} f^{\prime}\left(x_{i}\right) h_{i, j}\left(x_{i}, 0+\right) p_{i}\left(x_{i}\right) \mathrm{d} x_{i} \\
& +\frac{1}{2} f^{\prime}(0) \hat{a}_{i, i}(0+) p_{i}(0+)+\bar{\lambda}_{i} \int_{\mathbb{R}_{+}} \int_{-x_{i}}^{\infty}\left(f\left(x_{i}+z_{i}\right)-f\left(x_{i}\right)\right) K_{i}\left(x_{i}, \mathrm{~d} z_{i}\right) p_{i}\left(x_{i}\right) \mathrm{d} x_{i},
\end{aligned}
$$

where each operator $\hat{\mathcal{A}}_{i}$ is defined the same as $\mathcal{A}_{i}$ but with $b_{i}$ and $a_{i, i}$ replaced correspondingly by $\hat{b}_{i}$ and $\hat{a}_{i, i}$.

Theorem 4. The stationary density $p$ is in product form if and only if, for each $i, j \in\{1, \ldots, n\}$, $i \neq j$, we have

$$
\hat{a}_{i, j}\left(x_{i}, x_{j}\right)=\frac{h_{j, i}\left(x_{j}, 0+\right) p_{i}(0+)}{2 p_{i}\left(x_{i}\right)} \int_{x_{i}}^{\infty} p_{i}(\varsigma) \mathrm{d} \varsigma+\frac{h_{i, j}\left(x_{i}, 0+\right) p_{j}(0+)}{2 p_{j}\left(x_{j}\right)} \int_{x_{j}}^{\infty} p_{j}(\varsigma) \mathrm{d} \varsigma
$$

for $P_{i, j}$-a.e. $\left(x_{i}, x_{j}\right) \in \mathbb{R}_{+}^{2}$, where $P_{i, j}$ denotes, as in Theorem 3, the joint (stationary) law of the tuple $\left(X_{t}^{i}, X_{t}^{j}\right)$ in $\mathbb{R}_{+}^{2}$.

Remark 6. Note that if $\gamma$ and $R$ are nonrandom then $\hat{a}_{i, j}\left(x_{i}, x_{j}\right)=a_{i, j}\left(x_{i}, x_{j}\right)$ and

$$
h_{i, j}\left(x_{i}, 0+\right)=\left(\frac{R_{i, j}\left(x_{i}, 0\right)}{R_{j, j}(0)}\right) a_{j, j}(0+) .
$$

Therefore, in this case, even if the drift coefficients $\left\{b_{i}\right\}_{i=1}^{n}$ are random, the product-form condition in Theorem 4 takes the same form as in Theorem 3, but of course involving different one-dimensional marginal densities in the cases of random versus nonrandom $b$.

\section{Acknowledgements}

This research was supported in part by grants from the National Science Foundation (NSF), grant number ANI 0087404, and the Natural Sciences and Engineering Research Council of Canada (NSERC) through the discovery grant program, France Telecom, and CONICYT, Chile, FONDECYT project 1070797. 


\section{References}

[1] Bardhan, I. (1995). Further applications of a general rate conservation law. Stoch. Process. Appl. 60, 113-130.

[2] Gamarnik, D. and Zeevi, A. (2006). Validity of heavy traffic steady state approximations in open queueing networks. Ann. Appl. Prob. 16, 56-90.

[3] Harrison, J. M. (1985). Brownian Motion and Stochastic Flow Systems. John Wiley, New York.

[4] Harrison, J. M. and Reiman, M. I. (1981). Reflected Brownian motion in an orthant. Ann. Prob. 9, $302-308$.

[5] Harrison, J. AND Williams, R. J. (1987). Brownian models of open queueing networks with homogeneous customer populations. Stochastics 22, 77-115.

[6] Kella, O. And Whitt, W. (1990). Diffusion approximations for queues with server vacations. Adv. Appl. Prob. 22, 706-729.

[7] Konstantopoulos, T., Last, G. And Lin, S.-J. (2004). On a class of Lévy stochastic networks. Queueing Systems 46, 409-437.

[8] Kushner, H. J. (2001). Heavy Traffic Analysis of Controlled Queueing and Communication Networks (Appl. Math. 47). Springer, New York.

[9] Mazumdar, R. R. And Guillemin, F. M. (1996). Forward equations for reflected diffusions with jumps. Appl. Math. Optimization 33, 81-102.

[10] Piera, F., Mazumdar, R. and Guillemin, F. (2005). On product-form stationary distributions for reflected diffusions with jumps in the positive orthant. Adv. Appl. Prob. 37, 212-228.

[11] Piera, F., Mazumdar, R. and Guillemin, F. (2006). On the local times and boundary properties of reflected diffusions with jumps in the positive orthant. Markov Process. Relat. Fields 12, 561-582.

[12] Protter, P. (1990). Stochastic Integration and Differential Equations: A New Approach (Appl. Math. 21). Springer, New York.

[13] Reiman, M. I. (1984). Open queueing networks in heavy traffic. Math. Operat. Res. 9, 441-458.

[14] Reiman, M. I. and Williams, R. J. (1988). A boundary property of semimartingale reflecting Brownian motions. Prob. Theory Relat. Fields 77, 87-97.

[15] Rong, S. (2000). Reflecting Stochastic Differential Equations with Jumps and Applications (Research Notes Math. 408). Chapman \& Hall/CRC, London.

[16] Shen, X., Chen, H., DaI, J. G. And DaI, W. (2002). The finite element method for computing the stationary distribution of an SRBM in a hypercube with applications to finite buffer queueing networks. Queueing Systems Theory Appl. 42, 33-62.

[17] Whitт, W. (2001). The reflection map with discontinuities. Math. Operat. Res. 26, 447-484.

[18] WhitT, W. (2002). Stochastic-Process Limits. Springer, New York.

[19] Williams, R. J. (1995). Semimartingale reflecting Brownian motions in the orthant. In Stochastic Networks (IMA Vol. Math. Appl. 71), Springer, New York, pp. 125-137.

[20] Williams, R. J. (1998). Reflecting diffusions and queueing networks. In Proceedings of the International Congress of Mathematicians (Berlin, 1998), Vol. III, pp. 321-330 (electronic). 